\title{
The degree distribution in unlabelled 2-connected graph families
}

\author{
Veronika Kraus $\|^{\| t}$ \\ ${ }^{1}$ Institute of Discrete Mathematics and Geometry, TU Wien, Wiedner Hauptstraße 8-10/104, 1040 Wien
}

We study the random variable $X_{n}^{k}$, counting the number of vertices of degree $k$ in a randomly chosen 2-connected graph of given families. We prove a central limit theorem for $X_{n}^{k}$ with expected value $\mathbb{E} X_{n}^{k} \sim \mu_{k} n$ and variance $\mathbb{V} X_{n}^{k} \sim \sigma_{k}^{2} n$, both asymptotically linear in $n$, for both rooted and unrooted unlabelled 2-connected outerplanar or series-parallel graphs.

Keywords: unlabelled graphs, cycle index sums, central limit law

\section{1 introduction}

An outerplanar graph is a planar graph which can be embedded in the plane such that all nodes lie on the outer face. A series-parallel graph is a graph which is the result of series (subdivision) or parallel (doubling) extensions of the edges of a forest. (Both graph families can be described via families of forbidden minors $\left\{K_{4}, K_{23}\right\}$ and $\left\{K_{4}\right\}$, respectively, where $K_{4}$ denotes the complete graph on 4 vertices and $K_{23}$ denotes the complete bipartite graph with 2 vertices of one and 3 vertices of the second color).

Both the family of outerplanar and the family of series-parallel graphs are block-stable families of graphs, that is, a graph belongs to the family iff all of it's 2-connected components belong to the family. Blockstable families admit a decomposition into blocks and vertices (cf. Figure 1 ).

Further, both families mentioned above are so-called subcritical graph families. This are block-stable families where all 2-connected components in the decomposition are asymptotically of comparable and small size. In contrast, there are other families where a giant maximal block appears, which contains almost all nodes and the other components are of sublinear size (this phenomenon appears e.g. in planar graphs [14]). More examples for subcritical families are cacti graphs or graphs with a finite number of 3 -connected components.

A lot of work has been done recently on labelled block stable families, starting with the enumeration of labelled outerplanar, series-parallel [4] and planar graphs [11]. Results on the degree distribution are known for labelled outerplanar and series-parallel graphs [2, 9, 13], and also some weak results for all

\footnotetext{
${ }^{\dagger}$ Supported by the Austrian Science Foundation FWF, Project S9604.

1365-8050 @ 2010 Discrete Mathematics and Theoretical Computer Science (DMTCS), Nancy, France
} 

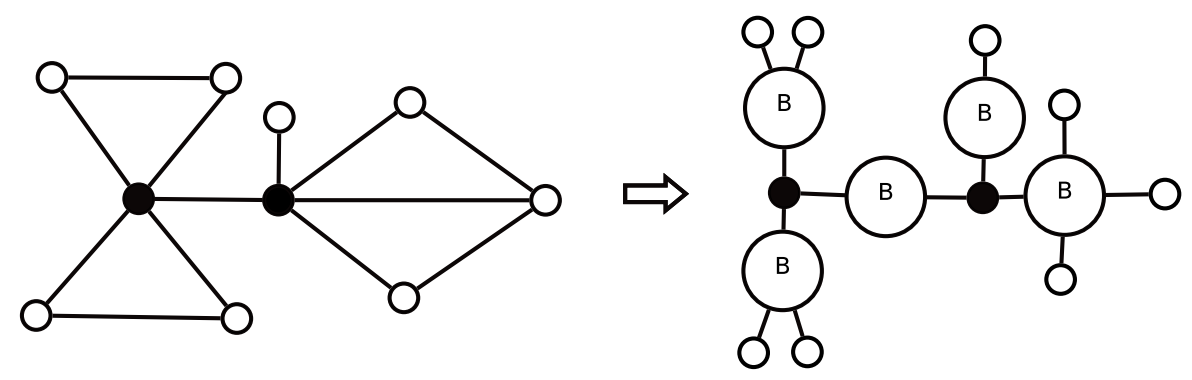

Fig. 1: An example for the decomposition of an outerplanar graph - the black and white dots denote vertices while the circles labelled with $B$ denote 2 -connected components

planar graphs [8]. In the more involved unlabelled framework, where symmetries make enumeration more difficult, less is known. Unlabelled outerplanar graphs have been counted by Fusy et al. in [3], general subcritical families are asymptotically counted in [7], where partial results on the degree distribution are also obtained. In this paper, we focus on maximal 2-connected components of outerplanar and seriesparallel graphs. We study the distribution of degrees among the vertices of 2-connected outerplanar and series-parallel graphs, which completes the distributional results presented in [7]. Let $\mathcal{B}$ be the family of 2connected components of either unlabelled outerplanar or series-parallel graphs. We prove the following theorem:

Theorem 1 The random variable that counts the number of vertices of degree $k$ in a randomly chosen member of $\mathcal{B}, X_{n}^{k}$, satisfies a central limit theorem with expected value $\mathbb{E} X_{n}^{k}=\mu_{k} n+\mathcal{O}(1)$ and variance $\mathbb{V} X_{n}^{k}=\sigma_{k}^{2} n+\mathcal{O}(1)$, where $\mu_{k}$ and $\sigma_{k}$ are real constants.

A corresponding result holds for connected and all unlabelled outerplanar and series-parallel graphs, and even for any block-stable subcritical family, without further knowledge on the degree distribution in their 2-connected components, which is proven in [7]. This is due to the fact that a connected blockstable graph $C$ is built by many (almost independent) 2-connected components, such that the number of vertices of degree $k$ in $C$ corresponds to a sum of weakly dependant random variables, that is, the sum of the numbers of vertices of degree $k$ in each of its blocks. This suggests a central limit theorem. For 2-connected graphs, there is no such decomposition into "smaller" components, therefore they have to be treated separately and it is a priori not clear that a central limit theorem will hold. However, it holds in the outerplanar and series-parallel case.

\section{Methods and Approach}

We consider unlabelled classes of graphs, both general families $\mathcal{G}$ as well as their derived families $\mathcal{G}^{\prime}$. The derived family $\mathcal{G}^{\prime}$ is the family of graphs in $\mathcal{G}$ where one vertex is distinguished (rooted) and is not counted. In order to obtain results on the degree distribution we refine the procedure of counting graph families, taking into account vertices of given degree. For this purpose, we use a generating functions approach. In the labelled case, families $\tilde{\mathcal{G}}$ are counted via exponential generating functions $\tilde{G}(x)=\sum_{n \geq 1} \tilde{g}_{n} \frac{x^{n}}{n !}$, where $\tilde{g}_{n}$ is the number of objects of size $n$ in $\tilde{\mathcal{G}}$. The exponential generating function of the derived 
family is then given by

$$
\tilde{G}^{\prime}(x)=\frac{\partial}{\partial x} \tilde{G}(x) .
$$

In the unlabelled framework, we have to take into account all symmetries arising from permutations on the set of vertices. Therefore, in order to obtain ordinary generating functions $G(x)=\sum_{n \geq 1} g_{n} x^{n}$ which count unlabelled families, we have to work with cycle index sums, a theory going back to Pólya, see e.g. [12]. Let $Z T(\sigma)=s_{1}^{\lambda_{1}} s_{2}^{\lambda_{2}} \cdots$ be the cycle type of a permutation $\sigma$, that is, $\sigma$ contains $\lambda_{i}$ cycles of length $i$ for $i \geq 1$. Then, the cycle index sum of a combinatorial class $\mathcal{G}$ is given by

$$
Z_{\mathcal{G}}\left(s_{1}, s_{2}, \ldots\right)=\sum_{G \in \mathcal{G}} \frac{1}{\left|\mathfrak{S}_{G}\right|} \sum_{\sigma \in \mathfrak{S}_{G}} Z T(\sigma)
$$

where $\mathfrak{S}_{G}$ is the set of permutations on the vertices of the object $G \in \mathcal{G}$. The cycle index sum of the derived family $\mathcal{G}^{\prime}$ is given by

$$
Z_{\mathcal{G}^{\prime}}\left(s_{1}, s_{2}, \ldots\right)=\frac{\partial}{\partial s_{1}} Z_{\mathcal{G}}\left(s_{1}, s_{2}, \ldots\right)
$$

The ordinary generating functions can be obtained from the cycle index sums by substituting $s_{i}=x^{i}$ : $G(x)=Z_{\mathcal{G}}\left(x, x^{2}, x^{3}, \ldots\right)$. Note that $\tilde{G}(x)=Z_{\mathcal{G}}(x, 0,0, \ldots)$.

To obtain our results, we will use multivariate generating functions

$$
B\left(x, v_{1}, v_{2}, \ldots, v_{k}, v_{\infty}\right)=\sum_{n, n_{1}, \ldots, n_{k}, n_{\infty}} b_{n, n_{1}, \ldots, n_{k}, n_{\infty}} x^{n} v_{1}^{n_{1}} \cdots v_{k}^{n_{k}} v_{\infty}^{n_{\infty}}
$$

where $b_{n, n_{1}, \ldots, n_{k}, n_{\infty}}$ is the number of unlabelled 2-connected graphs with $n=n_{1}+\cdots+n_{k}+n_{\infty}$ vertices, where $n_{\ell}$ vertices have degree $\ell, 1 \leq \ell \leq k$, and $n_{\infty}$ vertices have degree greater than $k$.

Therefore, we need to define cycle index sums for multiple variables $s_{i}, u_{j, i}, i \geq 1, j=1, \ldots, k, \infty$, where $s_{i}$ counts cycles of ordinary vertices while $u_{j, i}$ counts cycles of vertices with given degree $j$. To obtain the multivariate generating function, we substitute $s_{i}=x^{i}$ and $u_{j, i}=v_{j}^{i}$ for $i \geq 1$.

Our first goal will be to set up a core system of functional equations $\mathbf{y}(x, \mathbf{v})=\mathbf{F}(x, \mathbf{y}, \mathbf{v})$ where $\mathbf{y}(x, \mathbf{v})=\left(y_{1}(x, \mathbf{v}), \ldots, y_{N}(x, \mathbf{v})\right)$ and $\mathbf{v}=\left(v_{1}, \ldots, v_{k}, v_{\infty}\right)$, using combinatorial decompositions, such that the generating function of blocks is determined by a functional relation $B(x, \mathbf{v})=R(x, \mathbf{y}(x, \mathbf{v}), v)$. A powerful help in counting combinatorial structures is the following

Theorem 2 (dissymetry Theorem on trees(cf. [1])) Let $\mathcal{T}$ be a family of unrooted trees, and let $\mathcal{T}_{\circ}$ be the corresponding family of trees rooted at a vertex, $\mathcal{T}_{\circ-\circ}$ the family rooted at an edge and $\mathcal{T}_{\circ \rightarrow \circ}$ the family rooted at an oriented edge. Then there exists a bijection such that:

$$
\mathcal{T}+\mathcal{T}_{\circ \rightarrow \circ}=\mathcal{T}_{\circ}+\mathcal{T}_{\circ-\circ}
$$

Our second goal is to interpret the functions generated in the combinatorial part as analytic functions, examining their singular behaviour. We can then apply results from singularity analysis (see e.g. [6, 10]) to obtain asymptotic results such as a limiting degree distribution. Suppose that the system obtained in the combinatorial part is analytic and positive, that is, all coefficients are nonnegative and $\mathbf{F}(x, \mathbf{0}, \mathbf{v}) \neq 0$, and that it is strongly connected, that is, no subsystem can be solved before the whole system can be 
solved. Then, under suitable analytic conditions, a general result (Drmota-Lalley-Woods Theorem, see [6. Theorem 2.33]) proves that all $y_{i}(x, \mathbf{v})$ allow representations of the form

$$
y_{j}(x, \mathbf{v})=g_{j}(x, \mathbf{v})-h_{j}(x, \mathbf{v}) \sqrt{1-\frac{x}{\rho(\mathbf{v})}},
$$

where $g_{j}(x, \mathbf{v})$ and $h_{j}(x, \mathbf{v})$ are analytic functions around $\mathbf{v}=1$ and $x=\rho(\mathbf{1})$.

In general, we call a local representation $y(x, \mathbf{v})=g(x, \mathbf{v})-h(x, \mathbf{v})\left(1-\frac{x}{\rho(\mathbf{v})}\right)^{\alpha}$ a local singular expansion of $y(x, \mathbf{v})$ of order $\alpha$ for $\alpha \notin \mathbb{N}$.

Given a generating function $y(x, \mathbf{v})=\sum y_{n}(v) x^{n}$, which allows a singular expansion of order $\alpha \notin \mathbb{N}$, a well-known result (a corollary of the quasi power theorem, see [6, Theorem 2.35]) shows that a random variable which fulfills

$$
\mathbb{E} v^{X_{n}}=\frac{y_{n}(v)}{y_{n}(1)},
$$

satisfies a central limit theorem

$$
\frac{1}{\sqrt{n}}\left(X_{n}-\mathbb{E} X_{n}\right) \stackrel{d}{\rightarrow} N\left(0, \sigma^{2}\right)
$$

with $\mathbb{E} X_{n}=\mu n+\mathcal{O}(1)$ and $\mathbb{V} X_{n}=\sigma^{2} n+\mathcal{O}(1)$, where

$$
\mu=-\frac{\rho^{\prime}(1)}{\rho(1)}, \quad \sigma^{2}=-\frac{\rho^{\prime \prime}(1)}{\rho(1)}+\mu^{2}+\mu .
$$

\section{Outerplanar graphs}

As mentioned before, outerplanar graphs are planar graphs which can be embedded in the plane such that all nodes lie on the outerface. In this section, we will prove Theorem 1 for the family of 2-connected unlabelled outerplanar graphs $\mathcal{B}$. As a byproduct, we also obtain a central limit theorem for the derived family $\mathcal{B}^{\prime}$ :

Theorem 3 Let $\mathcal{B}$ be the family of random 2-connected unlabelled outerplanar graphs and $\mathcal{B}^{\prime}$ be it's derived family. Then the random variable that counts the number of vertices of degree $k$ in a randomly chosen member of $\mathcal{B}, X_{n}^{k}$, and the corresponding random variable $X_{n}^{\prime k}$ for a member of the derived family, satisfy a central limit theorem with expected value $\mathbb{E} X_{n}^{k} \sim \mathbb{E} X_{n}^{\prime k} \sim \mu_{k} n$ and variance $\mathbb{V} X_{n}^{k} \sim \mathbb{V} X_{n}^{\prime k} \sim$ $\sigma_{k}^{2} n$ where $\mu_{k}=2(k-1)(\sqrt{2}-1)^{k}$ and $\sigma_{k}^{2}$ is a computable constant.

Proof: As indicated in Section 2, the proof is divided into a combinatorial and an analytic part. Our first goal is to find a core system of functional equations with suitable properties for a subfamily of 2-connected outerplanar graphs. This system will lead to a local singular expansion for the bivariate generating function $B^{\prime(k)}(x, v)$ of rooted 2-connected graphs, where $v$ counts vertices of degree $k$. Analytic integration will lead to the result for the unrooted class.

\subsection{Combinatorial part}

Note that a 2-connected outerplanar graph can be interpreted as a dissection of a polygon. We set up a system of functional equations for the cycle index sums of oriented outer-edge rooted dissections, whose 
automorphism group consists of the identity only.

An oriented outer-edge rooted dissection $A^{o}$ can be decomposed in the following way: Considering the (inner) face containing the root edge, which is either a triangle or a $k$-gon, with $k>3$. If it is a triangle, the 2 edges other than the root edge are either single edges or can be considered as root-edges of other oriented outer-edge rooted dissections, the orientation of the edge is implied by the orientation of the root edge of the dissection. If $k>3$, we insert a virtual edge connecting the endpoint of the root edge with the starting point of the edge connecting to the starting point of the root edge. This imaginary edge is root-edge of another oriented outer-edge rooted dissection, just as the remaining edge is either root-edge or outer-edge (cf. Figure 2).

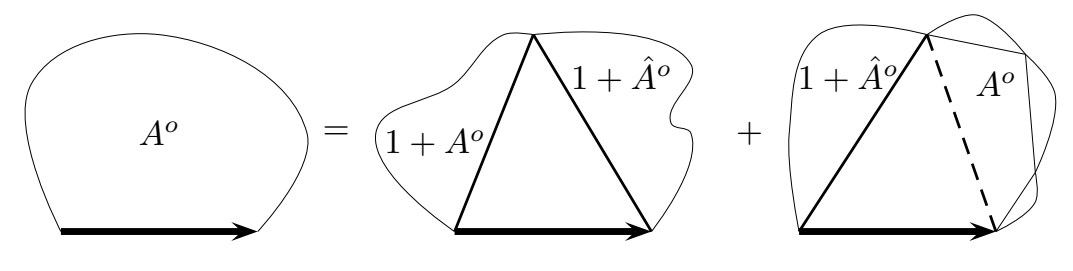

Fig. 2: decomposing an oriented outer-edge rooted dissection

Translating the above decomposition by the symbolic method (see e.g. [10]), we obtain the following system for the cycle index sums $Z_{\mathcal{A}_{i, j}^{o}}\left(\mathbf{s}_{\mathbf{1}} ; \mathbf{u}_{\mathbf{1}}\right)$ of oriented outer-edge rooted dissections, where the vertices of the root edge have degrees $i$ and $j$ for $i, j \in\{1, \ldots, k\}$ and degree greater than $k$ for $i, j=\infty$ and are not counted.

For brevity, we use the notation $\left(\mathbf{s}_{\mathbf{1}} ; \mathbf{u}_{\mathbf{1}}\right):=\left(s_{1}, u_{1,1}, \ldots, u_{k, 1}, u_{\infty, 1} ; s_{2}, u_{1,2}, \ldots, u_{k, 2}, u_{\infty, 2} ; \ldots\right)$. Note that $Z_{\mathcal{A}_{1,1}^{o}}\left(\mathbf{s}_{\mathbf{1}} ; \mathbf{u}_{\mathbf{1}}\right)=1, Z_{\mathcal{A}_{1, j}^{o}}\left(\mathbf{s}_{\mathbf{1}} ; \mathbf{u}_{\mathbf{1}}\right)=0$ for $j \neq 1$ and $Z_{\mathcal{A}_{i, j}^{o}}\left(\mathbf{s}_{\mathbf{1}} ; \mathbf{u}_{\mathbf{1}}\right)=Z_{\mathcal{A}_{j, i}^{o}}\left(\mathbf{s}_{\mathbf{1}} ; \mathbf{u}_{\mathbf{1}}\right)$ for $1 \leq i, j \leq k$ or $i, j=\infty$.

Lemma 4 The functions $Z_{\mathcal{A}_{i, j}^{o}}\left(\mathbf{s}_{\mathbf{1}} ; \mathbf{u}_{\mathbf{1}}\right)$ fulfill the following strongly connected system of equations:

$$
\begin{aligned}
Z_{\mathcal{A}_{i, j}^{o}}\left(\mathbf{s}_{\mathbf{1}} ; \mathbf{u}_{\mathbf{1}}\right)= & \sum_{l_{1}+l_{2} \leq k} u_{l_{1}+l_{2}, 1} Z_{\mathcal{A}_{i-1, l_{1}}^{o}}\left(\mathbf{s}_{\mathbf{1}} ; \mathbf{u}_{\mathbf{1}}\right) Z_{\mathcal{A}_{j-1, l_{2}}^{o}}\left(\mathbf{s}_{\mathbf{1}} ; \mathbf{u}_{\mathbf{1}}\right) \\
& +s_{1} u_{\infty, 1}\left(\sum_{l_{1}+l_{2}>k} Z_{\mathcal{A}_{i-1, l_{1}}^{o}}\left(\mathbf{s}_{\mathbf{1}} ; \mathbf{u}_{\mathbf{1}}\right) Z_{\mathcal{A}_{j-1, l_{2}}^{o}}\left(\mathbf{s}_{\mathbf{1}} ; \mathbf{u}_{\mathbf{1}}\right)\right) \\
& +\sum_{l_{1}+l_{2} \leq k+1} u_{l_{1}+l_{2}-1,1} Z_{\mathcal{A}_{i-1, l_{1}}^{o}}\left(\mathbf{s}_{\mathbf{1}} ; \mathbf{u}_{\mathbf{1}}\right) Z_{\mathcal{A}_{j-1, l_{2}}^{o}}\left(\mathbf{s}_{\mathbf{1}} ; \mathbf{u}_{\mathbf{1}}\right) \\
& +s_{1} u_{\infty, 1}\left(\sum_{l_{1}+l_{2}>k+1} Z_{\mathcal{A}_{i-1, l_{1}}^{o}}\left(\mathbf{s}_{\mathbf{1}} ; \mathbf{u}_{\mathbf{1}}\right) Z_{\mathcal{A}_{j, l_{2}}^{o}}\left(\mathbf{s}_{\mathbf{1}} ; \mathbf{u}_{\mathbf{1}}\right)\right), \forall 2 \leq i \leq j \leq k+1
\end{aligned}
$$

Proof: A close look at the recursive description depicted in Figure 2 leads to this system of equations. Strong connectivity is given as every equation depends on $Z_{\mathcal{A}_{\infty, \infty}^{o}}\left(\mathbf{s}_{\mathbf{1}} ; \mathbf{u}_{\mathbf{1}}\right)$ and the equation for $Z_{\mathcal{A}_{\infty, \infty}^{o}}\left(\mathbf{s}_{\mathbf{1}} ; \mathbf{u}_{\mathbf{1}}\right)$ depends on all other variables.

From the above system, we can deduce generating functions $A_{i, j}^{o}(x, \mathbf{v}), \mathbf{v}=\left(v_{1}, \ldots, v_{k}, v_{\infty}\right)$, where the 
variable $x$ counts all vertices while variables $v_{i}, i \in\{1, \ldots, k\}$, count vertices of degree $i$ and $v_{\infty}$ counts vertices of degree greater than $k$, by substituting $s_{\ell}=x^{\ell}, u_{i, \ell}=v_{i}^{\ell}$.

We will further need the cycle index sum of symmetric outer-edge rooted dissections, where the automorphism group contains the identity and a reflection which fixes the root edge. They fulfill the following decomposition.

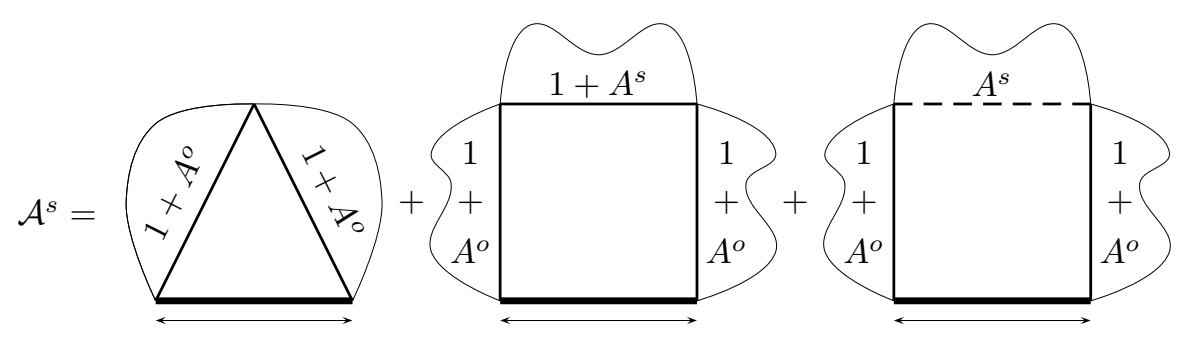

Fig. 3: The decomposition of a symmetric outer-edge rooted dissections

Analyzing the decomposition, we obtain a system of equations for the cycle index sums $Z_{A_{i, i}^{s}}\left(\mathbf{s}_{\mathbf{1}} ; \mathbf{u}_{\mathbf{1}}\right)$ of reflective dissections, where $Z_{\mathcal{A}_{i, i}^{s+}}\left(\mathbf{s}_{\mathbf{1}} ; \mathbf{u}_{\mathbf{1}}\right)$ represents the identity while $Z_{\mathcal{A}_{i, i}^{s-}}\left(\mathbf{s}_{\mathbf{1}} ; \mathbf{u}_{\mathbf{1}}\right)$ represents the reflecting part. Thus

$$
Z_{\mathcal{A}_{i, i}^{s}}\left(\mathbf{s}_{\mathbf{1}} ; \mathbf{u}_{\mathbf{1}}\right)=\frac{Z_{\mathcal{A}_{i, i}^{s+}}\left(\mathbf{s}_{\mathbf{1}} ; \mathbf{u}_{\mathbf{1}}\right)+Z_{\mathcal{A}_{i, i}^{s-}}\left(\mathbf{s}_{\mathbf{1}} ; \mathbf{u}_{\mathbf{1}}\right)}{2}
$$

For shorter notation, we use $\sum_{\ell=1}^{k, \infty} f(\ell)$ to denote the $\operatorname{sum} \sum_{\ell=1}^{k} f(\ell)+f(>k)$ and $\left(\mathbf{s}_{\ell} ; \mathbf{u}_{\ell}\right)$ to denote the set of variables $\left(s_{\ell}, u_{1 ; \ell}, \ldots, u_{\infty ; \ell} ; s_{2 \ell}, u_{1 ; 2 \ell}, \ldots, u_{\infty ; 2 \ell} ; \ldots\right)$. In $\left(\mathbf{s}_{\ell}^{\mathbf{r}}, \mathbf{u}_{\ell}^{\mathbf{r}}\right)$ every member of the set of variables is taken to the power $r:\left(s_{\ell}^{r}, u_{1, \ell}^{r}, \ldots, u_{\infty, \ell}^{r} ; \ldots\right)$. Note that indices arising through the constructions below which are greater than $k$ correspond to index $\infty$ and that for the summand with index $i=\infty$, index $\infty-1$ means index $\geq k$ (that is $k$ and $\infty$ ).

$$
\begin{aligned}
Z_{\mathcal{A}_{i, i}^{s+}}\left(\mathbf{s}_{\mathbf{1}} ; \mathbf{u}_{\mathbf{1}}\right)= & s_{1} \sum_{l=1}^{k, \infty}\left(Z_{\mathcal{A}_{i-1, l}^{o}}\left(\mathbf{s}_{\mathbf{1}}^{\mathbf{2}} ; \mathbf{u}_{\mathbf{1}}^{\mathbf{2}}\right) u_{2 l ; 1}\right) \\
& +s_{1}^{2}\left(\sum_{l=1}^{k, \infty} Z_{\mathcal{A}_{i-1, l}^{o}}\left(\mathbf{s}_{\mathbf{1}}^{\mathbf{2}} ; \mathbf{u}_{\mathbf{1}}^{\mathbf{2}}\right) \cdot\left(\sum_{l=1}^{k, \infty} u_{l+i ; 1}^{2} Z_{\mathcal{A}_{i, i}^{s+}}\left(\mathbf{s}_{\mathbf{1}} ; \mathbf{u}_{\mathbf{1}}\right)\right)\right) \\
& +s_{1}^{2}\left(\sum_{l=1}^{k, \infty} Z_{\mathcal{A}_{i-1, l}^{o}}\left(\mathbf{s}_{\mathbf{1}}^{\mathbf{2}} ; \mathbf{u}_{\mathbf{1}}^{\mathbf{2}}\right) \cdot\left(\sum_{i=2}^{k, \infty} z_{l+i-1 ; 1}^{2} Z_{A_{i, i}^{s+}}\left(\mathbf{s}_{\mathbf{1}} ; \mathbf{u}_{\mathbf{1}}\right)\right)\right)
\end{aligned}
$$




$$
\begin{aligned}
Z_{\mathcal{A}_{i, i}^{s-}}\left(\mathbf{s}_{\mathbf{1}} ; \mathbf{u}_{\mathbf{1}}\right)= & s_{1} \sum_{l=1}^{k, \infty}\left(Z_{\mathcal{A}_{i-1, l}^{o}}\left(\mathbf{s}_{\mathbf{2}} ; \mathbf{u}_{\mathbf{2}}\right) u_{2 l ; 1}\right) \\
& +s_{2}\left(\sum_{l=1}^{k, \infty} Z_{\mathcal{A}_{i-1, l}^{o}}\left(\mathbf{s}_{\mathbf{2}} ; \mathbf{u}_{\mathbf{2}}\right) \cdot\left(\sum_{l=1}^{k, \infty} u_{l+i ; 2} Z_{\mathcal{A}_{i, i}}^{s-}\left(\mathbf{s}_{\mathbf{1}} ; \mathbf{u}_{\mathbf{1}}\right)\right)\right) \\
& +s_{2}\left(\sum_{l=1}^{k, \infty} Z_{\mathcal{A}_{i-1, l}^{o}}\left(\mathbf{s}_{\mathbf{2}} ; \mathbf{u}_{\mathbf{2}}\right) \cdot\left(\sum_{i=2}^{k, \infty} z_{l+i-1 ; 2} Z_{\mathcal{A}_{i, i}^{s-}}\left(\mathbf{s}_{\mathbf{1}} ; \mathbf{u}_{\mathbf{1}}\right)\right)\right) .
\end{aligned}
$$

With the appropriate substitution, we obtain generating functions $A_{i}^{s}(x, \mathbf{v})$.

Let $\mathcal{B}_{i}^{\prime o}$ denote oriented vertex rooted dissections and $\mathcal{B}_{i}^{\prime s}$ denote reflective vertex-rooted dissections, both rooted at a vertex of degree $i$. With the help of $\mathcal{A}_{i, j}^{o}$ and $\mathcal{A}_{i, i}^{s}$ we obtain equations for the cycle index sums $Z_{\mathcal{B}_{i}^{\prime o}}\left(\mathbf{s}_{\mathbf{1}} ; \mathbf{u}_{\mathbf{1}}\right)$ and $Z_{\mathcal{B}_{i}^{\prime s}}\left(\mathbf{s}_{\mathbf{1}} ; \mathbf{u}_{\mathbf{1}}\right)$ :

$$
\begin{aligned}
& Z_{\mathcal{B}_{i}^{\prime o}}\left(\mathbf{s}_{\mathbf{1}} ; \mathbf{u}_{\mathbf{1}}\right)=s_{1} \sum_{j=1}^{k, \infty} u_{j, 1} Z_{\mathcal{A}_{i j}^{o}}\left(\mathbf{s}_{\mathbf{1}} ; \mathbf{u}_{\mathbf{1}}\right), \\
& Z_{\mathcal{B}_{i}^{\prime s-}}\left(\mathbf{s}_{\mathbf{1}} ; \mathbf{u}_{\mathbf{1}}\right)= \begin{cases}s_{1} \sum_{l=1}^{k+1} u_{2 l-1 ; 1} Z_{\mathcal{A}_{m, l}^{o}}\left(\mathbf{s}_{\mathbf{2}} ; \mathbf{u}_{\mathbf{2}}\right) & \text { for } i=2 m-1 \\
s_{2}\left(\sum _ { l = 1 } ^ { k + 1 } Z _ { \mathcal { A } _ { m , l } ^ { o } } ( \mathbf { s } _ { \mathbf { 2 } } ; \mathbf { u } _ { \mathbf { 2 } } ) \cdot \left(\sum_{j=1}^{k+1} Z_{\mathcal{A}_{j, j}^{s-}}\left(\mathbf{s}_{\mathbf{1}} ; \mathbf{u}_{\mathbf{1}}\right) u_{j+l ; 2}\right.\right. & \\
\left.\left.+\sum_{j=2}^{k+1} Z_{\mathcal{A}_{j, j}^{s-}}\left(\mathbf{s}_{\mathbf{1}} ; \mathbf{u}_{\mathbf{1}}\right) u_{j+l-1 ; 2}\right)\right) & \text { for } i=2 m .\end{cases}
\end{aligned}
$$

Finally, we obtain for the cycle index sum of rooted unlabelled 2-connected outerplanar graphs

$$
Z_{\mathcal{B}^{\prime}}\left(\mathbf{s}_{\mathbf{1}}, \mathbf{u}_{\mathbf{1}}\right)=\sum_{i=1}^{k, \infty} u_{i, 1} \frac{Z_{\mathcal{B}_{i}^{\prime o}}\left(\mathbf{s}_{\mathbf{1}} ; \mathbf{u}_{\mathbf{1}}\right)+Z_{\mathcal{B}_{i}^{\prime s-}}\left(\mathbf{s}_{\mathbf{1}} ; \mathbf{u}_{\mathbf{1}}\right)}{2}
$$

and, again by substitution, we obtain a functional equation for the generating function $B^{\prime}(x, \mathbf{v})$.

\subsection{Analytic part}

In a first step we analyze the core system (2) established in the previous part in terms of generating functions $\mathbf{A}^{\mathbf{0}}=\mathbf{F}\left(x, \mathbf{A}^{\mathbf{o}}, \mathbf{v}\right), \mathbf{A}^{\mathbf{o}}=\left(A_{i, j}^{o}\right)_{i, j \in\{1, \ldots, k, \infty\}}$ to obtain information on the singular behaviour. We obtain a distributional result on the derived family $\mathcal{B}^{\prime}$ from there, which we can extend to the unrooted family $\mathcal{B}$ by analytic integration.

Note that

$$
\sum_{i=1}^{k, \infty} \sum_{j=1}^{k, \infty} A_{i, j}^{o}(x, \mathbf{1})=A^{o}(x)
$$


is the ordinary generating function of oriented outer-edge rooted dissections with arbitrary root degree. We know from [3] that $A^{o}(x)$ has singularity $\rho=3-2 \sqrt{2}$ and a local singular expansion of order $\frac{1}{2}$ around it: $A^{o}(x)=g^{o}(x)-h^{o}(x) \sqrt{1-\frac{x}{\rho}}$. As the system of equations (2) is strongly connected and positive, all functions $A_{i, j}^{o}(x, 1)$ have the same singularity. Theorem [6, 2.33], as mentioned in Section 2 leads to a local singular expansion of order $\frac{1}{2}$ for the generating functions $A_{i, j}^{o(k)}(x, v)$, where only vertices of degree $k$ are counted:

$$
\begin{aligned}
A_{i, j}^{o(k)}(x, v) & =A_{i, j}^{o}(x, 1, \ldots, 1, v, 1) \\
& =g_{i j}(x, v)-h_{i j}(x, v) \sqrt{1-\frac{x}{\rho(v)}},
\end{aligned}
$$

with $\rho(1)=3-2 \sqrt{2}$. We further know that

$$
\sum_{i=1}^{k, \infty} A_{i, i}^{s}(x, \mathbf{1})=A^{s}(x)
$$

has radius of convergence $\sqrt{\rho}>\rho$. In the system of equations $(3)$ functions $A_{i, j}^{o}$ only appear in variables $s_{j}^{k}$ with exponents $k \geq 2$ and by strong connectivity and positivity, the same is true for every function $A_{i, i}^{s}(x, \mathbf{1})$, and thus, also

$$
A_{i, i}^{s(k)}(x, v)=A_{i, i}^{s}(x, 1 \ldots, 1, v, 1)
$$

has radius of convergence larger than $\rho(v)$ for $v$ sufficiently close to 1 .

We are interested in the number of nodes of degree $k$ in a member of $\mathcal{B}^{\prime}$ chosen uniformly at random, $X_{n}^{k}$. The probability, that a random graph of size $n$ has $m$ vertices of degree $k$ is given by $\frac{\left[x^{n} v^{m}\right] B^{\prime}(x, v)}{\left[x^{n}\right] B^{\prime}(x)}$, where $\left[x^{n}\right]$ denotes the coefficient of $x^{n}$ in the generating function. Thus, $X_{n}^{k}$ is of the form

$$
\mathbb{E} u^{X_{n}^{k}}=\frac{\left[x^{n}\right] B^{\prime}(x, v)}{\left[x^{n}\right] B^{\prime}(x, 1)} .
$$

The local singular expansion of $B^{\prime}(x, v)$ of order $\frac{1}{2}$ leads to a central limit theorem for the derived family $\mathcal{B}^{\prime}$ by means of Theorem [6, 2.35], as mentioned in Section 2 .

To obtain a result on the unrooted family, we will use integration. Therefore we isolate variable $s_{1}$ and count vertices of degree $k$, obtaining a trivariate generating function:

$$
B_{i}^{\prime(k)}(s, x, v)=Z_{\mathcal{B}_{i}^{\prime}}\left(s, 1, \ldots, 1, v, 1 ; x^{2}, 1, \ldots, 1, v^{2}, 1 ; x^{3}, 1, \ldots, 1, v^{3}, 1 ; \ldots\right) .
$$

Functions $B^{\prime(k)}(s, x, v)$ of rooted 2-connected outerplanar graphs with arbitrary root degree are then given by

$$
B^{\prime(k)}(s, x, v)=\sum_{i=1}^{k-1} B_{i}^{\prime(k)}(s, x, v)+v B_{k}^{(k)}(s, x, v)+B_{\infty}^{\prime(k)}(s, x, v) .
$$

As indicated in Section 2 the generating function of unrooted unlabelled 2-connected outerplanar graphs is then given by $B(x, v)=B(x, x, v)$, where

$$
B(s, x, v)=\int_{0}^{s} B^{\prime}(t, x, v) \mathrm{d} t+B(0, x, v) .
$$


It is important to note that $B(0, x, v)$ has bigger radius of convergence than $B^{\prime}(x, x, v)$ and thus has no influence on the asymptotic behaviour. $B(s, x, v)$ has a functional equations representation in terms of the core system (2) and (3), cf. Remark 5. The corresponding equations can be found in the Appendix. With these equations, it can be shown directly that $B(0, x, v)$ is analytic at $\rho(v)$.

Integration of $B^{\prime}(s, x, v)$ leads to a local singular expansion of order $\frac{3}{2}$

$$
B(s, x, v)=g(s, x, v)-h(s, x, v)\left(1-\frac{s}{\rho(x, v)}\right)^{\frac{3}{2}}
$$

It remains to prove that for $s=x, B(x, x, v)$ has a local singular expansion of order $\frac{3}{2}$ around it's singularity $\rho(v)$. If we set $s=x$, the singular term can be rewritten to

$$
\left(1-\frac{x}{\rho(x, v)}\right)^{\frac{3}{2}}=\kappa(x, v)\left(1-\frac{x}{\rho_{2}(v)}\right)^{\frac{3}{2}},
$$

where $\kappa(x, v)$ and $\rho_{2}(v)$ are analytic. Since $x=\rho(x, v)$ if and only if $x=\rho_{2}(v)$, it follows that $\rho_{2}(v)=\rho(v)$ and $B(x, v)$ has a local singular expansion of order $\frac{3}{2}$. From this expansion, the central limit theorem follows.

The expected value $\mathbb{E} X_{n}^{k} \sim \mathbb{E} X_{n}^{\prime k} \sim \mu_{k} n$ is asymptotically given by $\mu_{k}=-\frac{\rho^{\prime}(1)}{\rho(1)}$. Since the singularity $\rho(v)$ is the same as in the labelled case, we can adopt the result from the labelled case [6, Chapter 9], where $\mu_{k}=2(k-1)(\sqrt{2}-1)^{k}$. This matching of the singularities is due to the fact that the core system of equations in the labelled and unlabelled case are exactly the same and thus yield the same singularity. The other terms appearing in the unlabelled equations are analytic at $\rho(v)$, as seen before, and have no influence on the asymptotic result.

This result implies that the symmetries arising in 2-connected outerplanar graphs through unlabelling are very few and hence do not influence the asymptotic result. In the connected case, this is no longer true, as an exponential number of symmetries appears which alters the singularity of generating functions (see [7]).

Remark 5 The step of analytic integration could be omitted by using the dissymetry theorem on trees and the fact that the dual graph of a dissected polygon is a tree. One would then need to set up functional equations for the generating functions of inner-edge and face rooted dissections, which can be decomposed combinatorially into oriented and symmetric outer-edge rooted dissections. In [3] this method is used for counting unlabelled outerplanar graphs. In the case of multivariate cycle index sums like we use to count vertex degrees, systems of equations become very large and it is hardly possible to prove that the singularity is of type $\left(1-\frac{x}{\rho(v)}\right)^{\frac{3}{2}}$. Still, as we need them to prove that the radius of convergence of $B(0, x, v)$ is large enough and for comparison, the corresponding systems of equations can be found in the Appendix.

\section{Series-Parallel Graphs}

In the case of unlabelled 2-connected series-parallel graphs, we can proceed in a similar manner and obtain an analogous result as in the previous section, also obtaining a central limit theorem on the derived family as a byproduct. 
Theorem 6 Let $\mathcal{B}$ be the family of random 2-connected unlabelled series-parallel graphs and $\mathcal{B}^{\prime}$ be it's derived family. Then the random variable that counts the number of vertices of degree $k$ in a randomly chosen member of $\mathcal{B}, X_{n}^{k}$, and the corresponding random variable $X_{n}^{\prime k}$ for a member of the derived family, satisfy a central limit theorem with expected value $\mathbb{E} X_{n}^{k} \sim \mathbb{E} X_{n}^{\prime k} \sim \mu_{k} n$ and variance $\mathbb{V} X_{n}^{k} \sim \mathbb{V} X_{n}^{\prime k} \sim$ $\sigma_{k}^{2} n$ where $\mu_{k}$ and $\sigma_{k}$ are computable constants.

Proof: The subfamily of 2-connected series-parallel graphs, which provides us with a core system of equations are series-parallel networks. A series-parallel network is obtained via series-parallel extensions of a single edge, thus it is a series-parallel graph with 2 distinguished nodes (the endpoints of the original edge), which we call the poles and which we denote by 0 and $\infty$.

Let's denote by $\mathcal{D}$ the set of series-parallel networks. We distinguish 2 types: Series networks $\mathcal{S}$, which have a series decomposition, and parallel networks $\mathcal{P}$, which have a parallel decomposition. A single edge is also considered a network, which is neither series nor parallel. For the generating functions $D_{i j}(x, \mathbf{v})$, $S_{i j}(x, \mathbf{v})$ and $P_{i j}(x, \mathbf{v})$ of networks with pole degrees $i$ and $j$ and $\bar{D}_{i}(x, \mathbf{v}), \bar{S}_{i}(x, \mathbf{v})$ and $\bar{P}_{i}(x, \mathbf{v})$ of symmetric networks where a pole-exchanging symmetry is applied, we obtain (strongly connected) systems of equations for $1<i<j$ (note that $D_{i j}=D_{j i}$ ), see Appendix, Systems (6) and (7). This systems will be the core system of the proof.

For 2-connected graphs we use a decomposition into series, parallel and 3-connected components (which do not exist in our case as $K_{4}$ is exluded as a minor) which goes back to Tutte [15] and is described in detail in [5]. This decomposition leads to a bipartite tree with nodes colored $\mathcal{R}$ and nodes colored $\mathcal{M}$, where $\mathcal{R}$ denotes ring components and $\mathcal{M}$ denotes multiedge components, both with at least 3 edges. Series-parallel graphs are then obtained by replacing the edges of those ring- and multiedge components by parallel and series networks, respectively.

This decomposition applies for the rooted class as well as for the unrooted class, with the only difference that ring and multiedge components are rooted or not. In both cases we obtain systems of equations in terms of the core system on networks.

We proceed as in the outerplanar case and set up systems for the rooted class and use integration for unrooting. Nevertheless, systems of equations both for rooted and unrooted unlabelled series-parallel graphs can be found in the Appendix. To obtain a system of equations for the generating function of 2-connected series-parallel graphs we apply dissymetry theorem (Theorem 2 ) to the bipartite tree and obtain

$$
\mathcal{B}^{\prime}=1+\mathcal{B}_{\mathcal{R}^{\prime}}+\mathcal{B}_{\mathcal{M}^{\prime}}-\mathcal{B}_{\mathcal{R} \mathcal{M}},
$$

where $\mathcal{B}_{\mathcal{R}^{\prime}}=\mathcal{R}^{\prime} \circ_{e}(\mathcal{D}-\mathcal{S})$ denotes rings whose edges are substituted by non-series networks $\mathcal{D}-\mathcal{S}$, $\mathcal{B}_{\mathcal{M}^{\prime}}=\mathcal{M}^{\prime} \circ_{e}(S)$ denotes multiedges whose edges are substituted by series networks $\mathcal{S}$ and at most one edge, and $\mathcal{B}_{\mathcal{R M}}=x \cdot \mathcal{P} \cdot \mathcal{S}$ denotes an $R-M$ tree rooted at an edge, that is at the intersection of a ring and multiedge component. The additional 1 counts the single edge which is also considered a 2 -connected component.

\subsection{Analytic part}

We can express the core system of equations for networks $[6]$ in terms of just one network type $\mathcal{D}, \mathcal{S}$ or $\mathcal{P}$. Then we can proceed as in the previous section and note that $\sum_{i=1}^{k, \infty} \sum_{j=1}^{k, \infty} D_{i j}(x, \mathbf{1})=D(x)$ is the ordinary generating function of unlabelled series-parallel networks. The same applies for $\sum_{i} \sum_{j} S_{i j}(x, \mathbf{1})=$ $S(x)$ and $\sum_{i} \sum_{j} P_{i j}(x, \mathbf{1})=P(x)$. 
We know from [7] that $D(x), S(x)$ and $P(x)$ have a singularity $0<\rho<1$ and a square-root singular expansion around it. Thus by the same arguments of [6. Theorem 2.33] all functions $D_{i j}(x, v)$, specialized to count only vertices of degree $k$, have square root singular expansions

$$
D_{i j}(x, v)=D_{i j}(x, 1, \ldots, 1, v, 1)=g_{i j}(x, v)-h_{i j}(x, v) \sqrt{1-\frac{x}{\rho(v)}}
$$

while $\bar{D}_{i}$ has bigger radius of convergence. $B^{\prime}(x, v)$ fulfills functional equations in terms of $D_{i j}$ and $\bar{D}_{i}$ for $i, j \in\{1, \ldots, k, \infty\}$, and thus has the same square root expansion. Again, the random variable $X_{n}^{k}$ counting the number of vertices of degree $k$ in a random unlabelled rooted 2-connected series-parallel graph of size $n$ is of the form:

$$
\mathbb{E} u^{X_{n}^{\prime k}}=\frac{\left[x^{n}\right] B^{\prime}(x, v)}{\left[x^{n}\right] B^{\prime}(x, 1)},
$$

and thus Theorem [6, 2.35] applies and leads us to a central limit theorem for the derived family $\mathcal{B}^{\prime}$ of unlabelled series-parallel graphs.

To obtain the result for the unrooted class, we will again use integration. We have to isolate variable $s_{1}$ and obtain trivariate generating functions $B^{\prime}(s, x, v)$. Then

$$
B(s, x, v)=\int_{0}^{s} B^{\prime}(t, x, v) \mathrm{d} t+B(0, x, v)
$$

and $B(x, v)=B(x, x, v)$.

We need to check that $B(0, x, v)$ has bigger radius of convergence than $B^{\prime}(x, x, v)$. Then Integration of $B^{\prime}(s, x, v)$ leads to a local singular expansion of order $\frac{3}{2}$ around $(B(\rho, 1), \rho(1), 1)$. By applying the same arguments as in the case of unlabelled outerplanar graphs, this expansion translates to a expansion around $(\rho(1), 1)$ at $s=x$. Thus, we obtain a central limit theorem for the class of unrooted unlabelled 2 -connected series-parallel graphs again by [6, Theorem 2.35].

Remark 7 In the series-parallel case, we cannot be sure that the expected value $\mathbb{E} X_{n}^{k}$ will be the same as in the labelled case, where it is asymptotically given by $c k^{-\frac{3}{2}} q^{k}$, with constants $c, q$. The reason for that is the different core system of equations, where cycle index sums of symmetric groups appear in contrary to the labelled system. Giving an explicit representation of $\mu_{k}$ would require numerical calculation on the singularity $\rho(v)$ of the core system 6 .

\section{References}

[1] F. Bergeron, G. Labelle, and P. Leroux. Combinatorial Species and Tree-Like Structures. Cambridge University Press, 1998.

[2] N. Bernasconi, K. Panagiotou, and A. Steger. The degree sequence of random graphs from subcritical classes. Combinatorics, Probability and Computing, 18(5):647-681, 2009. 
[3] M. Bodirsky, E. Fusy, M. Kang, and S. Vigerske. Enumeration of unlabeled outerplanar graphs. Electronic Journal of Combinatorics, 14(1, research paper 66), 2007.

[4] M. Bodirsky, O. Giménez, M. Kang, and M. Noy. Enumeration and limit laws for series-parallel graphs. European J. Combin., 28(8):2091-2105, 2007.

[5] G. Chapuy, E. Fusy, M. Kang, and B. Shoilekova. A complete grammar for decomposing a family of graphs into 3-connected components. Electron. J. Combin., 15(1):R148, 2008.

[6] M. Drmota. Random Trees. Springer Wien, 2009.

[7] M. Drmota, E. Fusy, M. Kang, V. Kraus, and J. Rue. Asymptotic study of subcritical graph families. SIAM Journal on Discrete Mathematics. submitted. Available at http://arxiv.org/abs/1003.4699.

[8] M. Drmota, O. Giménez, and M. Noy. Degree distribution in random planar graphs. submitted,

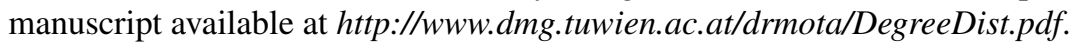

[9] M. Drmota, O. Giménez, and M. Noy. Vertices of given degree in series-parallel graphs. Random Structures Algorithms, 36(3):273-314, 2008.

[10] P. Flajolet and R. Sedgewick. Amalytic Combinatorics. Cambridge University Press, 2009.

[11] O. Giménez and M. Noy. Asymptotic enumeration and limit laws of planar graphs. J. Amer. Math. Soc., 22:309-329, 2009.

[12] F. Harary and E. Palmer. Graphical Enumeration. Academic Press New York and London, 1973.

[13] C. McDiarmid, A. Steger, and D. Welsh. Random planar graphs. J.Combin.Theory, (93):187-205, 2005.

[14] K. Panagiotou and A. Steger. Maximal biconnected subgraphs of random planar graphs. In Proceedings of the 20th Annual ACM-SIAM Symposium on Discrete Algorithms(SODA), pages 432-440, 2009.

[15] W. Tutte. Connectivity in graphs. Oxford U.P, 1966. 


\section{Appendix}

We state here equations not stated in the above proof as well as according figures.

\section{Missing equations in the outerplanar case}

As mentioned in Remark 1 in Section 3 the dual graph of a dissection of a polygon is a tree (cf Figure44. Vertex degrees are preserved by this duality, as the degree of a vertex in the dissection is equivalent to the distance between the 2 outer dual vertices neighbouring this vertex.

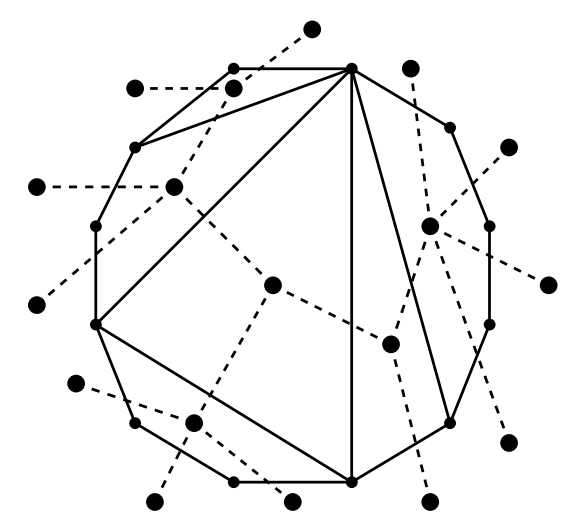

Fig. 4: The dual tree of a dissection

We use the dissymetry theorem (Theorem 2) to set up a system of equations for the multivariate cycle index sums $Z_{\mathcal{D}}\left(\mathbf{s}_{\mathbf{1}}, \mathbf{u}_{\mathbf{1}}\right)$ of unrooted unlabelled dissections, where the variables $\mathbf{s}_{\mathbf{1}}=s_{1}, s_{2}, \ldots$ count cycles of vertices while the variables $\mathbf{u}_{\mathbf{1}}=\left(u_{i, 1}, u_{i, 2}, \ldots\right)_{i \in\{1, \ldots, k, \infty\}}$ count cycles of vertices of degree $i$ for $i=1, \ldots, k$ and vertices of degree greater than $k$ for $i=\infty$. Translating the dissymetry theorem, we obtain

$$
\begin{aligned}
\mathcal{T} & =\mathcal{D} \\
\mathcal{T}_{\circ \rightarrow \circ} & =2 \mathcal{D}^{o}+2 \mathcal{D}^{i}-2 \mathcal{D}^{i(s)}+\mathcal{D}^{f(s)} \\
\mathcal{T}_{\circ} & =\mathcal{D}^{o}+\mathcal{D}^{f} \\
\mathcal{T}_{\circ-\circ} & =\mathcal{D}^{o}+\mathcal{D}^{i},
\end{aligned}
$$

where $\mathcal{D}$ denotes the family of unrooted dissections, $\mathcal{D}^{o}$ dissections rooted at an outer edge, $\mathcal{D}^{i}$ dissections rooted at an inner edge, $\mathcal{D}^{i(s)}$ dissections rooted at a symmetry edge, $\mathcal{D}^{f}$ dissections rooted at a face, $\mathcal{D}^{f(s)}$ rooted at a face containing a symmetry edge. Thus we obtain for $Z_{\mathcal{D}}$ :

$$
Z_{\mathcal{D}}=Z_{\mathcal{D}^{f}}-Z_{\mathcal{D}^{f(s)}}-Z_{\mathcal{D}^{i}}+2 Z_{\mathcal{D}^{i(s)}},
$$

In Section 3 we have set up systems of equations for oriented (2) and symmetric (3) outer-edge rooted dissections. We can use them to build systems for all other classes needed in the above equation. 
We use the same notation as in previous chapters and write $Z_{\mathcal{G}}\left(\mathbf{s}_{1}, \mathbf{u}_{1}\right)$ for the cycle index sum of a structure $\mathcal{G}$ with $\left(\mathbf{s}_{\mathbf{1}}, \mathbf{u}_{\mathbf{1}}\right)$ being the set of variables $\left(s_{1}, u_{1,1}, \ldots, u_{k, 1}, u_{\infty, 1} ; s_{2} \ldots\right)$ and $\left(\mathbf{s}_{\ell}, \mathbf{u}_{\ell}\right)$ denoting the modified set of variables $\left(s_{l}, u_{1, \ell}, \ldots, u_{k, \ell} u_{\infty, \ell} ; s_{2 \ell}, u_{1,2 \ell}, \ldots, u_{k, 2 \ell}, u_{\infty, 2 \ell} ; \ldots\right)$ for some $\ell \geq 2$.

Inner edge rooted dissections: Inner edge rooted dissections follow a decomposition into 2 outeredge rooted dissections, glued together at their root edge. Taking into account all symmetries, we obtain 4 classes of inner-edge rooted dissections, which we denote by $\mathcal{D}_{1}^{i}, \mathcal{D}_{2}^{i}, \mathcal{D}_{3}^{i}$ and $\mathcal{D}_{4}^{i}$ (cf. Figure 5 ):

This decomposition translates to the following system in the language of cycle indey sums:
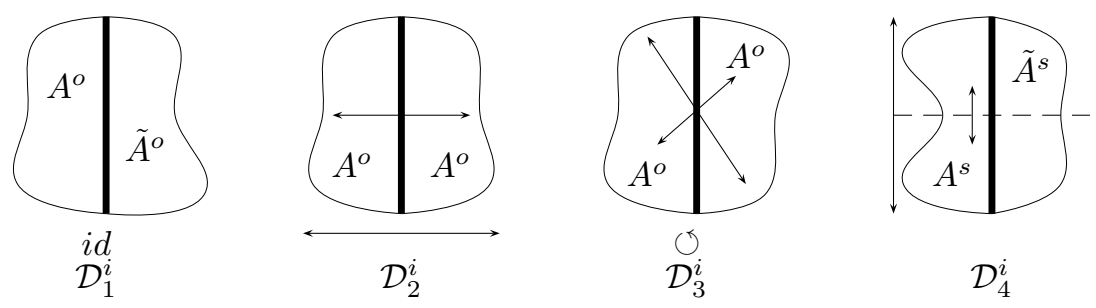

Fig. 5: Decomposition of inner edge rooted dissections

$$
\begin{aligned}
Z_{\mathcal{D}_{1}^{i}}\left(\mathbf{s}_{\mathbf{1}}, \mathbf{u}_{\mathbf{1}}\right) & =s_{1}^{2} \sum_{i_{1}=2}^{k, \infty} \sum_{j_{1}=2}^{k, \infty} \sum_{i_{2}=2}^{k, \infty} \sum_{j_{2}=2}^{k, \infty} u_{i_{1}+i_{2}-1 ; 1} u_{j_{1}+j_{2}-1 ; 1} Z_{\mathcal{A}_{i_{1}, j_{1}}^{o}}\left(\mathbf{s}_{\mathbf{1}}, \mathbf{u}_{\mathbf{1}}\right) Z_{\mathcal{A}_{i_{2}, j_{2}}^{o}}\left(\mathbf{s}_{\mathbf{1}}, \mathbf{u}_{\mathbf{1}}\right), \\
Z_{\mathcal{D}_{2}^{i}}\left(\mathbf{s}_{\mathbf{1}}, \mathbf{u}_{\mathbf{1}}\right) & =s_{1}^{2} \sum_{i=2}^{k, \infty} \sum_{j=2}^{k, \infty} u_{2 i-1 ; 1} u_{2 j-1 ; 1} Z_{\mathcal{A}_{i, j}^{o}}\left(\mathbf{s}_{\mathbf{2}} ; \mathbf{u}_{\mathbf{2}}\right), \\
Z_{\mathcal{D}_{3}^{i}}\left(\mathbf{s}_{\mathbf{1}}, \mathbf{u}_{\mathbf{1}}\right)= & s_{2} \sum_{i=2}^{k, \infty} \sum_{j=2}^{k, \infty} u_{i+j-1 ; 2} Z_{\mathcal{A}_{i, j}^{o}}\left(\mathbf{s}_{\mathbf{2}} ; \mathbf{u}_{\mathbf{2}}\right) \\
Z_{\mathcal{D}_{4}^{i}}\left(\mathbf{s}_{\mathbf{1}}, \mathbf{u}_{\mathbf{1}}\right)= & s_{2} \sum_{i_{1}=2}^{k, \infty} \sum_{i_{2}=2}^{k, \infty} u_{i_{1}+i_{2}-1 ; 2} Z_{\mathcal{A}_{i_{1}, i_{1}}^{s-}}\left(\mathbf{s}_{\mathbf{1}} ; \mathbf{u}_{\mathbf{1}}\right) Z_{\mathcal{A}_{i_{2}, i_{2}}^{s-}}\left(\mathbf{s}_{\mathbf{1}} ; \mathbf{u}_{\mathbf{1}}\right) \\
Z_{\mathcal{D}^{i}}\left(\mathbf{s}_{\mathbf{1}}, \mathbf{u}_{\mathbf{1}}\right)= & \frac{Z_{\mathcal{D}_{1}^{i}}\left(\mathbf{s}_{\mathbf{1}}, \mathbf{u}_{\mathbf{1}}\right)+Z_{\mathcal{D}_{2}^{i}}\left(\mathbf{s}_{\mathbf{1}}, \mathbf{u}_{\mathbf{1}}\right)+Z_{\mathcal{D}_{3}^{i}}\left(\mathbf{s}_{\mathbf{1}}, \mathbf{u}_{\mathbf{1}}\right)+Z_{\mathcal{D}_{4}^{i}}\left(\mathbf{s}_{\mathbf{1}}, \mathbf{u}_{\mathbf{1}}\right)}{4}
\end{aligned}
$$

Symmetric inner-edge rooted dissections consist of $\mathcal{D}_{2}^{i}, \mathcal{D}_{3}^{i}$ and $\mathcal{D}_{4}^{i}$ only.

Face rooted dissections: Let $l \geq 3$ be the size of the root face. Then, face rooted dissections follow a decomposition into outer-edge rooted dissections, as outer-edge rooted dissections are attached to the $l \geq 3$ edges of the root-face. Cycle index sums for face rooted dissections fulfill the following system of equations, where $Z_{\mathcal{F} o}\left(\mathbf{s}_{\mathbf{1}}, \mathbf{u}_{\mathbf{1}}\right)$ denotes oriented face rooted dissections (only cyclic permutations are allowed here), while $Z_{\mathcal{F}_{1}^{m}}\left(\mathbf{s}_{\mathbf{1}}, \mathbf{u}_{\mathbf{1}}\right), Z_{\mathcal{F}_{2}^{m}}\left(\mathbf{s}_{\mathbf{1}}, \mathbf{u}_{\mathbf{1}}\right)$ and finally $Z_{\mathcal{F}^{s}}\left(\mathbf{s}_{\mathbf{1}}, \mathbf{u}_{\mathbf{1}}\right)$ denote face rooted dissection where a reflection is applied. 


$$
\begin{aligned}
& Z_{\mathcal{F} o}\left(\mathbf{s}_{\mathbf{1}}, \mathbf{u}_{\mathbf{1}}\right)= \\
& =\sum_{l \geq 3} \frac{1}{l}\left(\sum_{d \mid l} \varphi(d)\left(\sum_{\substack{i_{1}, i_{2}, \ldots, i_{l} \frac{l}{d} \\
j_{1}, j_{2}, \ldots, j_{\frac{l}{d}}}} \prod_{m=1}^{\frac{l}{d}-1}\left(u_{i_{m+1}+j_{m}, d} u_{i_{\frac{l}{d}}+j_{1}, d} Z_{\mathcal{A}_{i_{m} j_{m}}^{o}}\left(\mathbf{s}_{\mathbf{d}}, \mathbf{u}_{\mathbf{d}}\right)\right) Z_{\mathcal{A}_{i_{\frac{l}{d}} j^{\frac{l}{d}}}}\left(\mathbf{s}_{\mathbf{1}}, \mathbf{u}_{\mathbf{1}}\right)\right)\right) .
\end{aligned}
$$

For $l=2 m+1$

$$
Z_{\mathcal{F}_{1}^{m}}\left(\mathbf{s}_{\mathbf{1}}, \mathbf{u}_{\mathbf{1}}\right)=s_{1} \sum_{i=0}^{k, \infty} Z_{\mathcal{A}_{i i}^{s-}}\left(\mathbf{s}_{\mathbf{1}}, \mathbf{u}_{\mathbf{1}}\right)\left(\sum_{\substack{i_{1}, i_{2}, \ldots, i_{m} \\ j_{1}, j_{2}, \ldots, j_{m}}} \prod_{t=1}^{m-1} u_{i+i_{1}, 2} u_{j_{t}+i_{t+1}, 2} u_{2 j_{m}} Z_{\mathcal{A}_{i_{t} j_{t}}^{o}}\left(\mathbf{s}_{\mathbf{2}}, \mathbf{u}_{\mathbf{2}}\right)\right)
$$

for $l=2 m+2$

$$
\begin{aligned}
& Z_{\mathcal{F}_{2}^{m}}\left(\mathbf{s}_{\mathbf{1}}, \mathbf{u}_{\mathbf{1}}\right)=s_{1}^{2}\left(\sum_{\substack{i_{1}, i_{2}, \ldots, i_{m+1} \\
j_{1}, j_{2}, \ldots, j_{m+1}}} \prod_{t=1}^{m} u_{2 i_{1}, 1} u_{j_{t}+i_{t+1}, 2} u_{2 j_{m+1}, 1} Z_{\mathcal{A}_{i_{t} j_{t}}^{o}}\left(\mathbf{s}_{\mathbf{2}}, \mathbf{u}_{\mathbf{2}}\right)\right) \\
& Z_{\mathcal{F}_{3}^{m}}\left(\mathbf{s}_{\mathbf{1}}, \mathbf{u}_{\mathbf{1}}\right)=\sum_{i=0}^{k, \infty} Z_{\mathcal{A}_{i i}^{s-}}\left(\mathbf{s}_{\mathbf{1}}, \mathbf{u}_{\mathbf{1}}\right) \sum_{\hat{i}=0}^{k, \infty} Z_{\mathcal{A}_{\hat{i} \hat{\imath}}^{s-}}\left(\mathbf{s}_{\mathbf{1}}, \mathbf{u}_{\mathbf{1}}\right) \\
& \times\left(\sum_{\substack{i_{1}, i_{2}, \ldots, i_{m} \\
j_{1}, j_{2}, \ldots, j_{m}}} \prod_{t=1}^{m-1} u_{i+i_{1}, 2} u_{j_{t}+i_{t+1}, 2} u_{j_{m}+\hat{i}, 2} Z_{\mathcal{A}_{i_{t} j_{t}}^{o}}\left(\mathbf{s}_{\mathbf{2}}, \mathbf{u}_{\mathbf{2}}\right)\right) \\
& Z_{\mathcal{F}^{s}}\left(\mathbf{s}_{\mathbf{1}}, \mathbf{u}_{\mathbf{1}}\right)=\sum_{m \geq 1} Z_{\mathcal{F}_{1}^{m}}\left(\mathbf{s}_{\mathbf{1}}, \mathbf{u}_{\mathbf{1}}\right)+Z_{\mathcal{F}_{2}^{m}}\left(\mathbf{s}_{\mathbf{1}}, \mathbf{u}_{\mathbf{1}}\right)+Z_{\mathcal{F}_{3}^{m}}\left(\mathbf{s}_{\mathbf{1}}, \mathbf{u}_{\mathbf{1}}\right) .
\end{aligned}
$$

\section{Missing equations in the Series-Parallel case}

For the sake of brevity, equations in this part are given in terms of ordinary generating functions, i.e. the substitution $s_{r} \leftarrow x^{r}, u_{i ; r} \leftarrow v_{i}^{r}$ has already been made. We denote by $\left(x^{\ell}, \mathbf{v}^{\ell}\right)=\left(x^{\ell}, v_{1}^{\ell}, \ldots, v_{k}^{\ell}, v_{\infty}^{\ell}\right)$. Core system for series-parallel networks for $1<i<j, i, j \in\{1, \ldots, k, \infty\}$ :

$\mathfrak{S}_{i}$ denotes the cycle index sum of the full symmetric group on $i$ elements and the notation $\mathfrak{S}_{i}[G(x, \mathbf{v})]$ 
denotes the substitution $s_{i} \leftarrow G\left(x^{i}, v_{1}^{i}, \ldots, v_{k}^{i}, v_{\infty}^{i}\right)$.

$$
\begin{aligned}
D_{i j}(x, \mathbf{v})= & S_{i j}(x, \mathbf{v})+P_{i j}(x, \mathbf{v}) \\
S_{i j}(x, \mathbf{v})= & x\left(\sum_{\ell_{1}=2}^{k, \infty} \sum_{\ell_{2}=1}^{k, \infty} v_{\ell_{1}+\ell_{2}} P_{i \ell_{1}}(x, \mathbf{v}) D_{\ell_{2} j}(x, \mathbf{v})\right) \\
P_{i j}(x, \mathbf{v})= & \sum_{\ell_{1}+2 \ell_{2}+\ldots+i \ell_{i}=i}\left(\sum_{\sum \in \mathfrak{S}_{i}:} \prod_{r=1}^{i} \mathfrak{S}_{l_{r}}\left[S_{r, \sigma(r)}(x, \mathbf{v})\right]\right) \\
& +\sum_{\ell_{1}+2 \ell_{2}=j}\left(\sum_{\substack{\sigma \in \mathfrak{S}_{i}: \\
\sigma(i) \ell_{\sigma(i)}=j}}^{i} \prod_{r=1}^{i} \mathfrak{S}_{l_{r}}\left[S_{r, \sigma(r)}(x, \mathbf{v})\right]\right) \\
& -\sum_{i=1}^{k, \infty} \sum_{j=1}^{k, \infty} S_{i j}(x, \mathbf{v})
\end{aligned}
$$

$$
\begin{aligned}
& \bar{D}_{i}(x, \mathbf{v})=\bar{S}_{i}(x, \mathbf{v})+\bar{P}_{i}(x, \mathbf{v}) \\
& \bar{S}_{i}(x, \mathbf{v})=\sum_{\ell=1}^{k, \infty}\left(D_{i \ell}\left(x^{2}, \mathbf{v}^{2}\right)\left(x v_{2 \ell}+x^{2} v_{\ell+1}^{2}+x \sum_{j=1}^{k, \infty} v_{\ell+j}^{2} \bar{P}_{j}(x, \mathbf{v})\right)\right. \\
& \bar{P}_{i}(x, \mathbf{v})=\sum_{\ell_{1}+2 \ell_{2}+\ldots+i \ell_{i}=i}\left(\prod_{r=1}^{i} \mathfrak{S}_{l_{r}}\left[s_{2 s-1} \leftarrow \bar{S}_{r}\left(x^{2 s-1}, \mathbf{v}^{2 s-1}\right), s_{2 s} \leftarrow S_{r r}\left(x^{2 s}, \mathbf{v}^{2 s}\right), s \geq 1\right]\right. \\
& \left.+\prod_{\substack{\ell_{t}=\ell_{\tilde{t}} \\
t \neq \tilde{t}}} \mathfrak{S}_{\ell_{t}}\left[S_{\ell_{t} \ell_{\tilde{t}}}\left(x^{2}, \mathbf{v}^{2}\right)\right]\right) \\
& +\sum_{\ell_{1}+2 \ell_{2}+\ldots+(i-1) \ell_{i-1}=i-1}\left(\prod_{r=1}^{i} \mathfrak{S}_{l_{r}}\left[s_{2 s-1} \leftarrow \bar{S}_{r}\left(x^{2 s-1} \mathbf{v}^{2 s-1}\right), s_{2 s} \leftarrow S_{r r}\left(x^{2 s}, \mathbf{v}^{2 s}\right), s \geq 1\right]\right. \\
& \left.+\prod_{\substack{\ell_{t}=\ell_{\tilde{t}} \\
t \neq \tilde{t}}} \mathfrak{S}_{\ell_{t}}\left[S_{\ell_{t} \ell_{\tilde{t}}}\left(x^{2}, \mathbf{v}^{2}\right)\right]\right)-\sum_{i=1}^{k, \infty} \bar{S}_{i}(x, \mathbf{v})
\end{aligned}
$$


For $i=1$ we have:

$$
\begin{array}{rlrl}
D_{11}(x, \mathbf{v}) & =1+S_{11}(x, \mathbf{v}), & & \\
D_{1 j}(x, \mathbf{v}) & =S_{1 j}(x, \mathbf{v}) & & \text { for } j>1 \\
\bar{D}_{1}(x, \mathbf{v}) & =1+\bar{S}_{1}(x, \mathbf{v}), & \\
S_{11}(x, \mathbf{v}) & =x v_{2}+x^{2}\left(\sum_{\ell_{1}=1}^{k, \infty} \sum_{\ell_{2}=1}^{k, \infty} v_{\ell_{1}+1} v_{\ell_{2}+1} D_{\ell_{1} \ell_{2}}(x, \mathbf{v})\right), & \\
S_{1 j}(x, \mathbf{v}) & =x\left(\sum_{\ell=1}^{k, \infty} v_{\ell+1} D_{\ell j}(x, \mathbf{v})\right) & & \text { for } j>1, \\
\bar{S}_{1}(x, \mathbf{v}) & =x v_{2}+x^{2}\left(\sum_{\ell=1}^{k, \infty} v_{\ell+1}^{2} \bar{D}_{i}(x, \mathbf{v})\right) . &
\end{array}
$$

Equations for rooted unlabelled 2-connected series-parallel graphs:

Remember that the cycle index sum of rooted 2-connected series parallel graphs is given by

$$
Z_{\mathcal{B}^{\prime}}\left(\mathbf{s}_{\mathbf{1}}, \mathbf{u}_{\mathbf{1}}\right)=1+Z_{\mathcal{B}_{\mathcal{R}^{\prime}}}\left(\mathbf{s}_{\mathbf{1}}, \mathbf{u}_{\mathbf{1}}\right)+Z_{\mathcal{B}_{\mathcal{M}^{\prime}}}\left(\mathbf{s}_{\mathbf{1}}, \mathbf{u}_{\mathbf{1}}\right)-Z_{\mathcal{B}_{\mathcal{R} \mathcal{M}}}\left(\mathbf{s}_{\mathbf{1}}, \mathbf{u}_{\mathbf{1}}\right)
$$

The same equation holds for generating functions:

$$
B^{\prime}(x, \mathbf{v})=1+B_{R^{\prime}}(x, \mathbf{v})+B_{M^{\prime}}(x, \mathbf{v})-B_{R M}(x, \mathbf{v})
$$

This leads to

$$
\begin{aligned}
& B_{R^{\prime}}(x, \mathbf{v})=x \sum_{i=1}^{k_{\infty}} v_{i} B_{R^{\prime} i}(x, \mathbf{v}) \\
& B_{R^{\prime} i}(x, \mathbf{v})=\frac{1}{2}\left[\sum_{i_{1}+j_{1}=i} \sum_{\ell \geq 3}\left(\sum_{\substack{i_{2}, i_{3}, \ldots, i_{\ell} \\
j_{2}, j_{3}, \ldots, j_{\ell}}}^{\ell-1} \prod_{r=1}^{\ell}\left(v_{i_{r}+j_{r}}(D-S)_{i_{r} j_{r+1}}(x, \mathbf{v})(D-S)_{i_{\ell} j_{1}}(x, \mathbf{v})\right)\right)\right. \\
& +\sum_{m \geq 1}\left(\sum_{\substack{i_{1}, i_{2}, \ldots, i_{m} \\
j_{1}, j_{2}, \ldots, j_{m}}} \prod_{r=1}^{m-1} v_{j_{r}+i_{r}}^{2} v_{i_{m} j_{m}}^{2}(D-S)_{\frac{i}{2} j_{1}}\left(x^{2}, \mathbf{v}^{2}\right)(D-S)_{i_{r} j_{r+1}}\left(x^{2}, \mathbf{v}^{2}\right)(\bar{D}-\bar{S})_{i_{m}}\right. \\
& \left.+x \sum_{\substack{i_{1}, i_{2}, \ldots, i_{m} \\
j_{1}, j_{2}, \ldots, j_{m+1}}} \prod_{r=1}^{m} v_{j_{r}+i_{r}}^{2} v_{2 j_{m+1}}^{2}(D-S)_{\frac{i}{2} j_{1}}\left(x^{2}, \mathbf{v}^{2}\right)(D-S)_{i_{r} j_{r+1}}\left(x^{2}, \mathbf{v}^{2}\right)\right]
\end{aligned}
$$




$$
\begin{aligned}
& B_{M^{\prime}}(x, \mathbf{v})=x \sum_{i=1}^{k_{\infty}} v_{i} B_{M^{\prime} i}(x, \mathbf{v}) \\
& B_{M^{\prime} i}(x, \mathbf{v})=x\left(\sum_{\ell_{1}+2 \ell_{2}+\ldots+i \ell_{i}=i}\left(\sum_{\sigma \in \mathfrak{S}_{i}} v_{\sum_{r=1}^{i} \sigma(i) \ell_{\sigma(i)}} \prod_{r=1}^{i} \mathfrak{S}_{l_{r}}\left[S_{r, \sigma(r)}(x, \mathbf{v})\right]\right)\right. \\
& +\sum_{\ell_{1}+2 \ell_{2}+\ldots+(i-1) \ell_{i-1}=i-1}\left(\sum_{\sigma \in \mathfrak{S}_{i}} v_{1+\sum_{r=1}^{i} \sigma(i) \ell_{\sigma(i)}} \prod_{r=1}^{i} \mathfrak{S}_{l_{r}}\left[S_{r, \sigma(r)}(x, \mathbf{v})\right]\right) \\
& -\sum_{j=1}^{k, \infty} S_{i j}(x, \mathbf{v}) \\
& B_{R M}(x, \mathbf{v})=\frac{1}{2} x\left(\sum_{\ell_{1}=1}^{k, \infty} \sum_{\ell_{2}=1}^{k, \infty} \sum_{\ell_{3}=1}^{k, \infty} \sum_{\ell_{4}=1}^{k, \infty} v_{\ell 1+\ell 2} v_{\ell_{3}+\ell_{4}} S_{\ell_{1} \ell_{3}}(x, \mathbf{v}) P_{\ell_{2} \ell_{4}}(x, \mathbf{v})\right) \\
& +\frac{1}{2} x\left(\sum_{\ell_{1}=1}^{k, \infty} \sum_{\ell_{2}=1}^{k, \infty} v_{\ell_{1}+\ell_{2}}^{2} \bar{S}_{\ell_{1}}(x, \mathbf{v}) \bar{P}_{\ell_{2}}(x, \mathbf{v})\right)
\end{aligned}
$$

Equations for unrooted unlabelled 2-connected series-parallel graphs:

The cycle index sum of unrooted 2-connected series parallel graphs is given by a similar equation. The only difference is that the ring- and multiedge components are no longer rooted:

$$
Z_{\mathcal{B}}\left(\mathbf{s}_{\mathbf{1}}, \mathbf{u}_{\mathbf{1}}\right)=1+Z_{\mathcal{B}_{\mathcal{R}}}\left(\mathbf{s}_{\mathbf{1}}, \mathbf{u}_{\mathbf{1}}\right)+Z_{\mathcal{B}_{\mathcal{M}}}\left(\mathbf{s}_{\mathbf{1}}, \mathbf{u}_{\mathbf{1}}\right)-Z_{\mathcal{B}_{\mathcal{R} \mathcal{M}}}\left(\mathbf{s}_{\mathbf{1}}, \mathbf{u}_{\mathbf{1}}\right) .
$$

Again, the equation can be translated into generating functions:

$$
B(x, \mathbf{v})=1+B_{R}(x, \mathbf{v})+B_{M}(x, \mathbf{v})-B_{R M}(x, \mathbf{v}),
$$

Also in this part, we will state equations in terms of generating functions. The function $B_{R M}(x, \mathbf{v})$ is nearly identical to the rooted one, with the only difference that we have to count the additional vertex:

$$
\begin{aligned}
B_{R M}(x, \mathbf{v})= & \frac{1}{2} x^{2}\left(\sum_{\ell_{1}=1}^{k, \infty} \sum_{\ell_{2}=1}^{k, \infty} \sum_{\ell_{3}=1}^{k, \infty} \sum_{\ell_{4}=1}^{k, \infty} v_{\ell 1+\ell 2} v_{\ell_{3}+\ell_{4}} S_{\ell_{1} \ell_{3}}(x, \mathbf{v}) P_{\ell_{2} \ell_{4}}(x, \mathbf{v})\right) \\
& +\frac{1}{2} x^{2}\left(\sum_{\ell_{1}=1}^{k, \infty} \sum_{\ell_{2}=1}^{k, \infty} v_{\ell_{1}+\ell_{2}}^{2} \bar{S}_{\ell_{1}}(x, \mathbf{v}) \bar{P}_{\ell_{2}}(x, \mathbf{v})\right)
\end{aligned}
$$


The other two generating functions, on the contrary, are more involved. This is due to the cyclic permutations and reflections that can be applied to the rings and multiedges now that they are unrooted:

$$
\begin{aligned}
& B_{R}(x, \mathbf{v})=\sum_{\ell \geq 3} \frac{1}{\ell} x^{\ell} \sum_{d \mid \ell} \varphi(d)\left(\sum_{\substack{i_{1}, i_{2}, \ldots, i_{\frac{\ell}{d}} \\
j_{1}, j_{2}, \ldots, j_{\frac{\ell}{d}}}} \prod_{r=1}^{\frac{\ell}{d}-1}\left(v_{j_{r}+i_{r+1}} v_{j_{\frac{\ell}{d}}+i_{1}}(D-S)_{i_{r} j_{r}}\left(x^{d}, \mathbf{v}^{d}\right)\right)\right) \\
& +\sum_{m \geq 1}\left(x \sum_{i=1}^{k, \infty} \sum_{\substack{i_{1}, i_{2}, \ldots, i_{m} \\
j_{1}, j_{2}, \ldots, j_{m}}} \prod_{r=1}^{m-1} v_{2 i_{1}} v_{j_{r}+i_{r+1}}^{2} u_{j_{r}+1}^{2}(D-S)_{i_{r} j_{r}}\left(x^{2}, \mathbf{v}^{2}\right)(\bar{D}-\bar{S})_{i}(x, \mathbf{v})\right. \\
& \left.+\sum_{i=1}^{k, \infty} \sum_{j=1}^{k, \infty} \sum_{\substack{i_{1}, i_{2}, \ldots, i_{m} \\
j_{1}, j_{2}, \ldots, j_{m}}} \prod_{r=1}^{m-1} v_{i+i_{1}}^{2} v_{j_{r}+i_{r+1}}^{2} v_{j+j_{m}}^{2}(D-S)_{i_{r} j_{r}}\left(x^{2}, \mathbf{v}^{2}\right)(\bar{D}-\bar{S})_{i}(\bar{D}-\bar{S})_{j}\right)(x, \mathbf{v}) \\
& \left.+x^{2} \sum_{\substack{i_{1}, i_{2}, \ldots, i_{m+1} \\
j_{1}, j_{2}, \ldots, j_{m+1}}} \prod_{r=1}^{m} v_{2 i_{1}}^{2} v_{j_{r}+i_{r+1}}^{2} v_{2 j_{m+1}}^{2}(D-S)_{i_{r} j_{r}}\left(x^{2}, \mathbf{v}^{2}\right)\right) \\
& B_{M}(x, \mathbf{v})=\sum_{i_{1}, i_{2}, \ldots, i_{k}, i_{\infty}} \sum_{\sigma \in \mathfrak{S}_{k+1}}\left(\left(v_{\sum_{\ell=1}^{k, \infty} \ell i_{\ell}} v_{\sum_{\ell=1}^{k, \infty} \sigma(\ell) j_{\sigma(\ell)}}+v_{\sum_{\ell=1}^{k, \infty} \ell i_{\ell}+1} v_{\sum_{\ell=1}^{k, \infty} \sigma(\ell) j_{\sigma(\ell)}+1}\right)\right. \\
& \left.\times \prod_{r=1}^{k, \infty} \mathfrak{S}_{\ell_{r}}\left[S_{r, \sigma(r)}(x, \mathbf{v})\right]\right) \quad-\left(v_{0}^{2}+v_{1}^{2}\right) \\
& -\sum_{i=1}^{k, \infty} \sum_{j=1}^{k, \infty}\left(v_{i} v_{j}+v_{i+1} v_{j+1}\right) S_{i j}(x, \mathbf{v})-\sum_{\substack{i_{1}, i_{2}, j_{1}, j_{2} \\
\in\{1, \ldots, k, \infty\}}} v_{i_{1}+i_{2}} v_{j_{1}+j_{2}} \frac{S_{i_{1} j_{1}}(x, \mathbf{v}) S_{i_{2} j_{2}}(x, \mathbf{v})+S_{i_{1} j_{1}}\left(x^{2}, \mathbf{v}^{2}\right)}{2} \\
& +\sum_{i_{1}, i_{2}, \ldots, i_{k}, i_{\infty}}\left(v_{\sum_{\ell=1}^{k, \infty} \ell i_{\ell}}^{2}+v_{\sum_{\ell=1}^{k, \infty} \ell i_{\ell}+1}^{2}\right) \\
& \times\left(\prod_{r=1}^{i} \mathfrak{S}_{l_{r}}\left[s_{2 s-1} \leftarrow \bar{S}_{r}\left(x^{2 s-1}, \mathbf{v}^{2 s-1}\right), s_{2 s} \leftarrow S_{r r}\left(x^{2 s}, \mathbf{v}^{2 s}\right), s \geq 1\right]\right. \\
& \left.+\prod_{\substack{\ell_{t}=\ell_{\tilde{t}} \\
t \neq \tilde{t}}} \mathfrak{S}_{\ell_{t}}\left[S_{\ell_{t} \ell_{\tilde{t}}}\left(x^{2}, \mathbf{v}^{2}\right)\right]\right) \\
& -\left(v_{0}+v_{1}\right)-\sum_{i=1}^{k, \infty}\left(v_{i}^{2}+v_{i+1}^{2}\right) \bar{S}_{i}(x, \mathbf{v})-\sum_{\substack{i_{1}, i_{2}, j_{1}, j_{2} \\
\in\{1, \ldots, k, \infty\}}} v_{i_{1}+i_{2}}^{2} \frac{\bar{S}_{i_{1}}(x, \mathbf{v}) \bar{S}_{i_{2}}(x, \mathbf{v})+S_{i_{1} i_{2}}\left(x^{2}, \mathbf{v}^{2}\right)}{2}
\end{aligned}
$$


